\title{
Right sided congenital diaphragmatic hernia, an operative challenge
}

\begin{abstract}
Congenital diaphragmatic hernia is one of the most challenging malformations associated with high mortality. Right side diaphragmatic hernia is rare $(20 \%)$ as compared to left side $(80 \%)$ with overall incidence of 1 in 5000 live births. Risk factors associated with poor outcome include low birth weight, prematurity, associated structural anomalies, and chromosomal defects. This is a case of isolated CDH diagnosed post natally, respiratory distress started immediately after birth, with herniation of Liver in to right hemi thorax, wide diaphragmatic defect, severe pulmonary hypertension and no structural defects. The successful Post natal management of this case with high frequency oscillatory ventilation and diligent operative technique, and institution of muscle paralysis (atracurium infusion) in the postoperative period has yielded optimum result in this case. The inpatient stay was for 25days.
\end{abstract}

Keywords: congenital diaphragmatic hernia, abdominal, tracheal, stabilization
Volume 2 Issue 3 - 2015

\author{
Abdulrahman Almaawi,' Prasad DRK,' \\ Zakaullah Waqasi,' Abdulla Alsharani, ${ }^{2}$ Ahmed \\ Aref, ${ }^{2}$ Ahmed Safwat ${ }^{2}$ \\ 'Department of Pediatric Surgery, King Abdullah Hospital, Saudi \\ Arabia \\ ${ }^{2}$ Neonatal intensive care unit, King Abdullah Hospital, Saudi \\ Arabia
}

Correspondence: Abdulrahman Almaawi, Director of Infection Prevention \& Control dept., Consultant pediatric surgeon, Department of Pediatric Surgery, King Abdullah Hospital, Po box 60, Bisha, Saudi Arabia,Tel 58I 505050, Fax 176209784, Emailalmaawi@yahoo.com

Received: April 12, 20I5 | Published: June 02, 2015
Abbreviations: $\mathrm{CDH}$, congenital diaphragmatic hernia; LHR, lung to head ratio; MRI, magnetic resonance imaging; HFOV, high frequency oscillatory ventilation; CT scan, computed tomography scan; NICU, neonatal intensive care unit; ECMO, extracorporeal membrane oxygenation

\section{Introduction}

Embryologically the diaphragm develops during the $8^{\text {th }}$ and $12^{\text {th }}$ week of gestation. Septum transversum separates the thoracic from abdominal cavity, later muscle fibres migrate in to this membrane. Left side defects are more common due to the late closure of this membrane. The loss of lung development can be explained by loss of space to develop, but other structural deficiencies are added bronchial, vessel and alveolar membrane deficiencies. Because the abdominal contents have migrated, the abdominal cavity will not grow as much. Then after surgical correction it may be difficult to reduce all organs in to abdominal cavity without pressure. Prenatal imaging provides valuable information. The diagnosis of $\mathrm{CDH}$ can be made prenatally by Ultrasonography in $90 \%$ of cases. Typically this is diagnosed at the $24^{\text {th }}$ week of gestation, but some have reported diagnosing it as early as $11^{\text {th }}$ week. Fetal ultrasound findings include polyhydramnios, bowel loops in the chest, echogenic chest mass.

Two distinct features have been used to risk stratification:

i. Low lung to head ratio (LHR)

ii. Liver herniation in to the chest.

LHR in accordance with age is well defined. LHR more than 1.35 is considered as borderline or high risk group at 28 weeks of gestation. Survival based on liver herniation alone is $43 \%$ as compared to $93 \%$ survival without herniation. Foetal MRI has been used as an adjunct to evaluation of foetal lung volume and liver herniation. Prenatal diagnosis of $\mathrm{CDH}$ provides an opportunity to optimize the prenatal and post natal care. Newborns with $\mathrm{CDH}$ present with respiratory distress, these infants will have a scaphoid abdomen and increased chest diameter. Bowel sounds can be heard in the chest. The diagnosis of $\mathrm{CDH}$ is made with ease by chest radiograph.
Initial post natal therapy is targeted at resuscitation and stabilization of infant in cardiopulmonary distress. In severe cases prompt endotracheal intubation is warranted. Initial mechanical ventilation should be utilized for stabilization. Pulmonary hypertension and associated cardiac anomalies are evaluated with echocardiography. The timing of operative procedure is based on clinical judgement and surgeon's discretion.

\section{Case report}

A newborn male with a gestational age of 38 weeks delivered by simple vaginal delivery, weighed $3.4 \mathrm{~kg}$, born with weak cry, dusky color, intubated and connected to ventilator (HFO ventilation was instituted with mean pressure of 13, FiO2 100\%, ABG PH 7.28, $\mathrm{PCO} 242.5, \mathrm{PO} 226.5, \mathrm{HCO} 3$ 18.4) his preductal $\mathrm{SPO} 295 \%$ and post ductal SPO2 $85 \%$ difference by 10 . The chest X-ray and CT scan has confirmed the presence of Right congenital diaphragmatic hernia. Echo has revealed severe pulmonary hypertension $(55-60 \mathrm{~mm})$. The rest of the investigations are normal. Neonate was taken up for Surgery after stabilization (Figure $1 \& 2$ ).

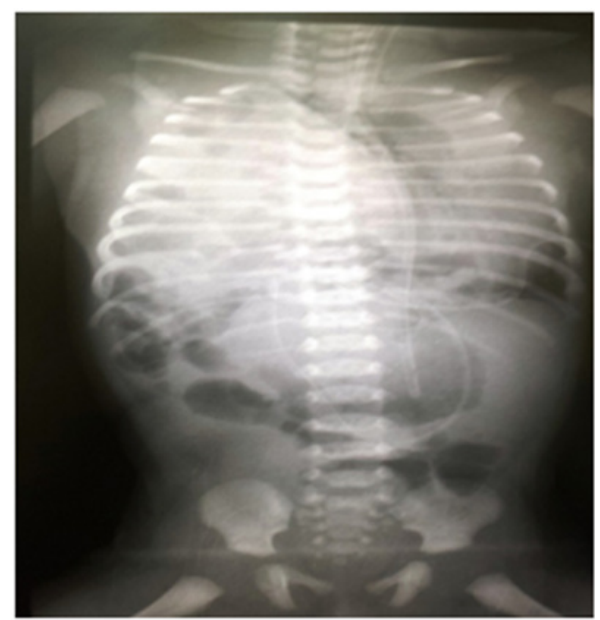

Figure I X-ray chest showing Right Congenital Diaphragmatic Hernia. 


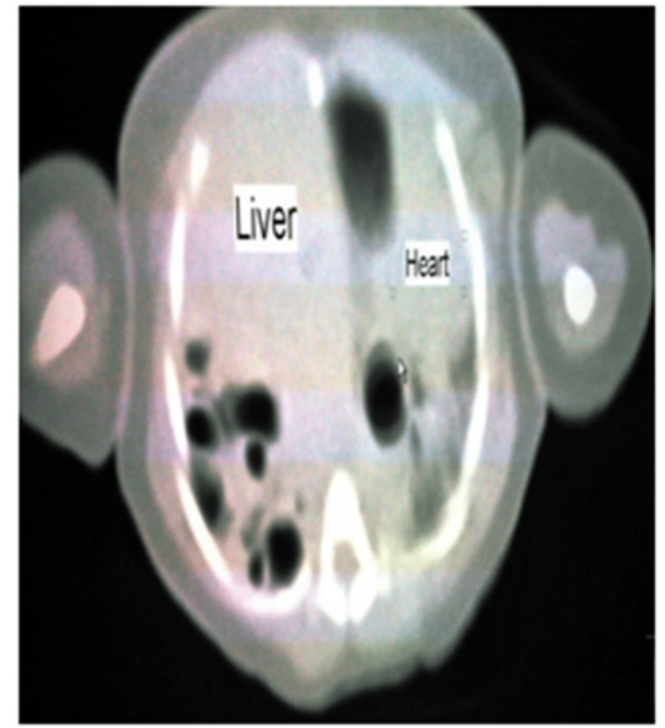

Figure 2 CT scan of chest showing liver at the level of heart.

Surgery was performed by right transverse abdominal incision. The entire liver was intra-thoracic along with intestinal loops. Liver was twisted by 90 degrees in the thoracic cavity. Stomach and Spleen were found in the abdomen. Only a thin rim of diaphragm on the anterior aspect was present, and the posterior rim was absent. The right lung was very small in size. The liver could be brought down to the abdomen with difficulty, and the intestinal loops were reduced. Repair of the diaphragm was achieved by placing sequential sutures through the anterior rim and the pleural flap. On lay gortex patch was kept. The abdomen was closed primarily without much tension. Post operatively baby was returned to NICU and kept on High Frequency Oscillatory Ventilation. Atracurium infusion was started and infant was kept paralyzed for initial four days. The baby was started on Nasogastric Feed on the fifth post operative day, initially half strength, and later full feeds. The chest $\mathrm{x}$ ray on the Seventh post operative day has shown partial expansion of right lung, and was weaned from ventilation after two weeks. The baby had a remarkable recovery and was discharged on twenty-fifth post operative day, and is under follow-up (Figure 3 \& 4).

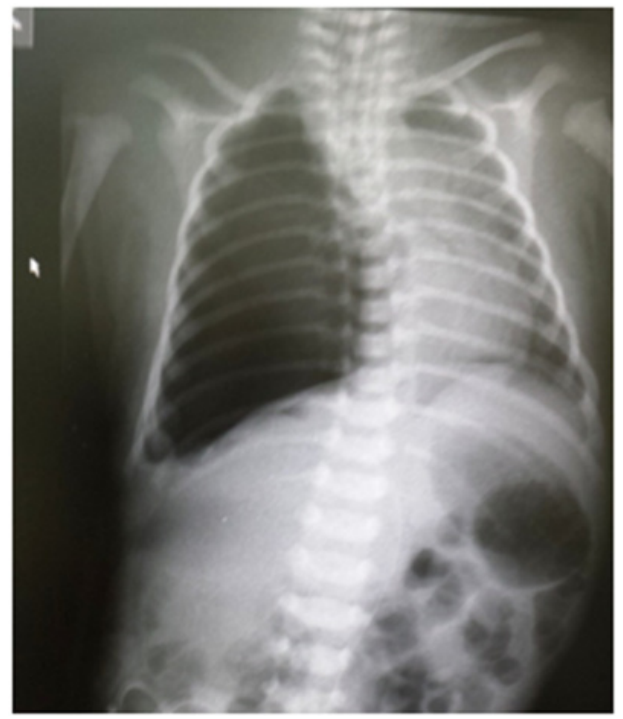

Figure 3 Chest $X$-ray on first post operative day with no intercostal drain.

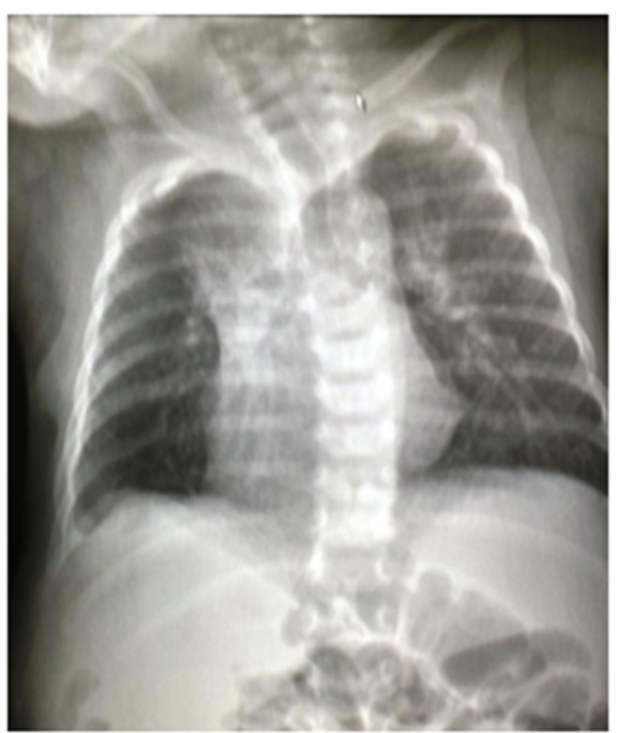

Figure 4 Chest $X$-ray before dishcarge showing good right lung expansion.

\section{Discussion}

Historically $\mathrm{CDH}$ was regarded a surgical emergency and the new born was rushed to the Operating Room for surgical correction. In 1987 Bohn et al., ${ }^{1}$ proposed a period of medical stabilization and delayed surgical repair in an attempt to improve the overall condition of the neonate. There is no prenatal diagnosis of $\mathrm{CDH}$ in this case, and the respiratory distress started from the time of birth which constitutes this to be a high risk case. Three prenatal features (liver herniation in to thorax, low lung to head ratio, and diagnosis before 25 weeks gestation) are likely to have a poor outcome with conventional treatment. ${ }^{2}$ In some centers these patients were offered antenatal intervention (tracheal occlusion) by foetoscopic methods. $\mathrm{CDH}$ interventions evolved from open fetal surgery to current state of endoscopic endoluminal tracheal occlusion. Currently the technique involves the placement of occlusion balloon, without maternal laparotomy or general anesthesia. Tracheal balloons are placed between 24 and 28 weeks of gestation and deflated at 34 weeks. ${ }^{3}$ The management of this high risk neonate has been according to the protocol of resuscitation, high frequency ventilation since birth, inhaled nitric oxide, and delayed surgical repair once the neonate is haemodynamically stable.

The surgical repair was difficult in this right sided $\mathrm{CDH}$ because the size of the defect. Was more than four centimeters. Only a thin rim of diaphragm was present anteriorly and the posterior rim was absent completely. The reduction of liver posed a difficult problem. Liver replacement in the abdomen can be complicated by kinking of hepatic veins causing profound hypotension. Potential anatomic anomalies such as possible hepatopulmonary fusion, ${ }^{4,5}$ anomalous venous drainage uniquely associated with right sided defects. Survival based on liver herniation alone is $43 \%$ as compared to $93 \%$ survival without liver herniation. ${ }^{6}$ The series published by Fischer et al., ${ }^{7}$ has shown the survival rate (right $\mathrm{CDH} 55 \%$ to left $\mathrm{CDH} 77 \%$ ) ECMO requirement (right $\mathrm{CDH} 40 \% \mathrm{Vs}$ left $\mathrm{CDH} 15 \%$ ) prosthetic material in R CDH Vs L CDH (76\% Vs 41\%) and abdominal wall (38\% Vs $19 \%)$ repairs. These data support that right side $\mathrm{CDH}$ carries a high mortality and morbidity. The repair of a $\mathrm{CDH}$ may be as variable as clinical management. A transverse incision on the ipsilateral side is our approach. Less than $10 \%$ surgeons prefer a thoracic approach. 
The type of repair is dependent on the size of the defect. If the defect is small, a tension free primary surgical closure should be performed with non-absorbable sutures. If the defect is wide primary closure may be attempted by one of the patch methods described below (Table 1).

Table I Repair options for large defects ${ }^{8}$

\begin{tabular}{|c|}
\hline Prosthesis \\
\hline Polypropylene (MARLEX) \\
\hline Polytetrafluoroethylene ( PTFE) (GORTEX) \\
\hline Dacron \\
\hline Muscle Flap \\
\hline Abdominal $^{9}$ \\
\hline Latissimus dorsi ${ }^{10}$ \\
\hline Latissimus dorsi and serratus anterior $"$ " \\
\hline Bioactive Material \\
\hline Surgisis ${ }^{12}$ \\
\hline
\end{tabular}

In this case we have used a gortex patch which we consider is much superior to biphasic or marlex mesh. Out comes regarding the prosthetic patch material are mixed. Currently the most popular material is small intestine sub mucosa (surgisis cook inc.) or human a cellular dermal matrix (alloderm, life cell). The bioactive material may act as a matrix for permanent tissue in growth. Long term outcomes of biosynthetic patches for $\mathrm{CDH}$ remain to be seen muscle flaps may be advantageous in very large defects and in diaphragmatic agenesis post operatively tube thoracostomy is probably not indicated. ${ }^{13}$ An exception may be $\mathrm{CDH}$ repair on ECMO because bleeding complication should be expected. The combination of entire liver herniation in to thorax, hypoplastic right lung, and near total absence of hemi diaphragm on the right side posed a difficult situation for the surgical team. Continuous infusion of atracurium is preferred in this case because respiratory or other muscular movements may jeopardize the success of repair. Weaning from the ventilator support was slow as tolerated by the infant. In patient stay for this neonate was 25 days compared with the total inpatient mean stay of 10 days. In spite of these challenges this neonate could be salvaged because of the efforts of neonatal Intensivists and timely intervention of the pediatric surgeons.

\section{Conclusion}

Preoperative physiological stabilization and subsequent elective repair has become the corner stone of management of $\mathrm{CDH}$ like our case. The introduction of a treatment protocol, using high frequency oscillatory ventilation since birth, inhaled nitric oxide if required, delayed surgical repair after stabilization improved the survival of this neonate with CDH. Success in this difficult case is optimized by the close cooperation between neonatologist, anesthetist and the pediatric surgeon. Though the hospital stay was prolonged the outcome regarding survival is excellent in this high risk case.

\section{Acknowledgments}

None.

\section{Conflicts of interest}

Author declares there are no conflicts of interest.

\section{Funding}

None.

\section{References}

1. Bohen D, Tamaura M, Perrin D Barker G, et al. Ventilatory predictictors of pulmonary hypoplasia in Congenital diaphragmatic hernia confirmed by morphologic assessment. J Pediatr . 1987;111(3): 423-431.

2. Harrison MR, Mychaliska GB, Albanese CT, et al. Correction of congenital Diaphragmatic hernia in utero IX: fetuses with poor prognosis (liver herniation and low lung-to-head ratio) can be saved by fetoscopic temporary tracheal Occlusion. J Pediatr Surg. 1998;33(7):1017-1023.

3. Taso K, Lally KP. Congenital diaphragmatic hernia and eventration. Ashcraft's pediatric surgery (5 ${ }^{\text {th }}$ edn). 210. p.304-321.

4. Olenik D, Codrich D, Gobbo F, et al. Hepatopulmonary fusion in a newborn. An uncommon intraoperatory Findings during right congenital diaphragmatic hernia surgery: Case description and review of literature. Hernia. 2014;18(3): 417-421.

5. Saurabh K, Kumar S, Chellani H, et al. Hepatic pulmonary fusion: a rare association of right sided congenital diaphragmatic hernia. Ann Gastroenterol . 2013;26(1): 95-96.

6. Albanese CT, Lopoo J, Goldstein RB, et al. Fetal liver position and perinatal outcome for congenital diaphragmatic hernia. Prenat Diagn. 1998;18(11):1138-1142

7. Fisher JC, Jefferson RA, Arkovitz MS, et al. Redefining outcomes in right congenital diaphragmatic hernia. J Pediatr Surg. 2008;43(2):373-379.

8. Clark RH, Hardin WD, Hirschl RB, et al. Current surgical management of Congenital diaphragmatic hernia: a report from the Congenital Diaphragmatic Hernia Study Group. J Pediatr Surg. 1998;33(7):1004-1009.

9. Joshi SB, Sen S, Chacko J, et al. Abdominal muscle flap repair for large defects of the diaphragm. Pediatr Surg Int. 2005;21(8):677-680.

10. Sydorak RM, Hoffman W, Lee H, et al. Reversed latissimus dorsi muscle flap for repair of recurrent congenital diaphragmatic hernia. J Pediatric Surg. 2003;38(3): 296-300.

11. Samarakkody U, Klaassen M, Nye B. Reonstruction of congenital agenesis of the hemidiaphragm by combined reverse lattismus dorsi and serratus anterior muscle flaps. J Pediatr Surg. 2001;36(11):1637-1640.

12. Grethel EJ, Cortes RA, Wagner AJ, et al. Prosthetic patches for congenital diaphragmatic hernia repair: Surgisis Vs Gore-Tex. J Pediatr Surg . 2006;41(1):29-33.

13. Wung JT, Sahni R, Moffitt ST, et al. Congenital diaphragmatic hernia: Survival treated with very delayed surgery, spontaneous respiration, and no chest tube. J Pediatr Surg. 1995;30(3):406-409. 